\title{
An experimental study on multi-slices CT angiography of the basilar artery in rabbit model of cerebral vasospasm
}

\author{
Rui $\mathrm{Li}^{1}$, Jinjin $\mathrm{Liu}^{1}$, Weijian Chen ${ }^{1, \text { a }}$, Yuxia Duan ${ }^{1}$, Yunjun Yang ${ }^{1}$, Zilong $\mathrm{Hu}^{1}$, \\ Xianxi Tan $^{2}$, Ming Zhong ${ }^{2}$ and Qichuan Zhuge ${ }^{2}$ \\ ${ }^{1}$ Department of Radiology/ Molecular and Digital Medical Imaging Institute, The First Affiliated \\ Hospital , Wenzhou Medical University, Wenzhou 325000 ,China. \\ ${ }^{2}$ Zhejiang Provincial Key Laboratory of Aging and Neurological Disorder Research, Wenzhou \\ 325000, China \\ ${ }^{a}$ Corresponding author: Weijian Chen, email: wyyycwj@163.com
}

Keywords: Vasospasm; Intracranial; Tomography; X-ray computed; Rabbit

\begin{abstract}
Background: Current methods for diagnosing cerebral vasospasm (CVS) include transcranial Doppler (TCD) ultrasonography and the current gold standard of digital subtraction angiography (DSA). The diagnosis of CVS is straightforward, but DSA is limited in its ability to quantify cerebral blood flow or the risk of cerebral ischemia leading to stroke. Sensitivity and specificity of TCD can vary greatly, depending on the level of experience of the operator. The purpose of this study was to explore the result of the multi-slices CT angiography (MSCTA) of the rabbit basal artery (BA), and to determine the value of MSCTA in rabbit model of CVS. Methods: In this study 25 Japan rabbits were randomly divided into two groups: control $(n=5)$ and subarachnoid hemorrhage (SAH) $(n=20)$. A rabbit basilar artery CVS model was developed by injection of autologous arterial blood into the cisterna rnagna twice, and non-ionic iodinated contrast medium (Omnipaque $350 \mathrm{mgI} / \mathrm{mL}$ ) was injected through posterior auricular central venous puncture at a dosage of $2.6 \mathrm{~mL} / \mathrm{kg}$ weight. High pressure syringe was used at an injection speed of $0.4 \mathrm{~mL} / \mathrm{s}$ and CT scan began with a delay time of 15 seconds. BA of the rabbit underwent MSCTA by GE Lightspeed pro CT scanner with imaging workstation ADW 4.3 edition. Source images were adopted by volume rendering (VR). Results: MSCTA of the rabbit BA were completed in 25 experiment rabbits, and the corresponding achievement ratio was $100 \%$. The mean transverse diameter of basilar artery in the rabbits measured by VR was $1.30 \mathrm{~mm}$. CVS occurred at day 1, and reached the peak at day 4. Basilar artery vasospasm was observed to have been improved moderately at day 11 . Conclusions: MSCTA can directly demonstrate the BA of rabbit and was a new tool for evaluation in vivo animal models of CVS.
\end{abstract}

\section{Introduction}

The most commonly used and available method of evaluating the degree of vasospasm is the conventional cerebral angiography or selective vertebral-basilar artery angiography technique [1]. In order to investigate diverse aspects of vasospasm, including its pathogenesis and the effect of drug as well as gene therapy, so many different in vitro and in vivo animal models of vasospasm have been developed in the domestic and overseas [2-5]. As there is no network between intracranial vascular and outside in the rabbit, the level of development of end to end anastomosis that in the brain branches of blood vessel observed in the rabbit models are similar to those observed in humans [6]. Therefore, rabbits become one of the most common alternative species of choice in cerebral vasospasm research. Since cerebral angiography performed on rabbits has certain difficulties, the present study was designed to show the basilar artery of the Japanese big-ear rabbit by multi-slices CT angiography (MSCTA) technique and thus intended to substitute for the production of rabbit model of vertebral - basilar artery angiography. 


\section{Material and Methods}

Experimental Animals and Grouping. All experiments were performed according to the protocol evaluated and approved by the Animal Care and Use Committees at Wenzhou Medical University, China. Twenty-five Japanese big-ear rabbits (provided by Laboratory Animal Center of Wenzhou Medical University), including both male and female, were from the same general level of animal origin with weight of 2.5 to $3.2 \mathrm{~kg}$. Each rabbit was numbered with black paint on the body. In this study, the rabbits underwent five times MSCTA before and after the experiment. Five rabbits were randomized to be the control group $(n=5)$ in which contrast agent may react on vascular stimulation, after the first MSCTA scanning. Others as the subarachnoid hemorrhage (SAH) model group $(n=20)$ in which fresh autologous arterial blood was injected into the cisterna magna of each rabbit after the first MSCTA30 60 minutes and the second injection was accomplished on day 1 (24 h after the first injection), using the same procedure as described above. Then two to five MSCTA examinations were performed in both of the two groups on day 1, 4, 7 and 11.

Methods of SAH Model. The rabbit was fixed on the operating table. The regional hair of the neck was sheared, and the skin was sterilized with $75 \%$ alcohol or iodine. Each animal in both experimental groups was anesthetized with an intramuscular injection of a mixture of ketamine (25 $\mathrm{mg} / \mathrm{kg}$ ).Sterile scissors were then used to make an incision through the occipital midline. The incision was about $2.5 \mathrm{~cm}$,by separating the atlantooccipital junction to the fascia, using sharp separation. Make sure the chin of the rabbit adduction, so that it could adequate access to craniocervical junction. An $18 \mathrm{G}$ trocar, at an angle of $30^{\circ}$ with the torso, was inserted through the skin inferior to the occipital protuberance. When the needle passed into the cisterna magna about $1 \mathrm{~cm}$, slight negative pressure was applied. The needle was kept in place until clear CSF flowing out. After the withdrawal of $0.5 \mathrm{ml} / \mathrm{kg}$ of the cerebrospinal fluid (CSF), $2.5 \mathrm{ml}$ non-heparinized fresh autologous auricular arterial blood taken from the central ear artery was injected into the cisterna magna by a slow injection. Then pulled out the trocar, sew up the incision after the local compression. The animals were then placed in a $30^{\circ}$ head-down tilted position for 15 min to ensure that the blood spread into the basal cistern. Twenty-four hours after the first $\mathrm{SAH}$, a second one was produced in the same manner as the first.

MSCTA Scanning and Post-processing Technique. All imaging was performed by using a LightSpeed multi-slice spiral CT scanner (GE Lightspeed pro). Image acquisition parameters were as follows: $120 \mathrm{kVp}$ and $210 \mathrm{~mA} ; 512 \times 512$ image matrix; 9.6cm field of view; The section thickness was $1.25 \mathrm{~mm}$, with a $0.5 \mathrm{~mm}$ overlap between successive sections; pitch of 0.562:1, and $0.8 \mathrm{~s}$ gantry rotation time. The scanning range of the rabbit was including entire head cover. The post aurem central veins was punctured by $24 \mathrm{G}$ trocar. Once the back blood appeared, connected the heparin lock and fixed it. Animals were placed in a prone position on the CT machine table. The NO.8 needle was used for linking heparin lock and high-pressure syringe. After positioning the scanning, non-ionic iodinated contrast medium (Omnipaque $350 \mathrm{mgI} / \mathrm{mL}$ ) was injected through posterior auricular central venous puncture at a dosage of $2.6 \mathrm{~mL} / \mathrm{kg}$ weight. High pressure syringe was used at an injection speed of $0.4 \mathrm{~mL} / \mathrm{s}$ and CT scan began with a delay time of 15 seconds. With imaging workstation ADW4.3 edition, source images was adopted by volume rendering(VR). In order to distinguish the basilar artery and skull, pseudo-color was used and the VR image could be magnified properly. By using VR to measure the diameter of the basilar artery at each time of the rabbits and pay more attention to the morphology of comparison. Measurements of basilar artery diameter contained within the selected proximal, middle, and distal artery segments, and the inner diameter of the basilar artery was calculated from the mean value of the diameters at these 3 sections. We defined diameter of the basilar artery, which before SAH as the baseline. Similarly, we defined diameter of the basilar artery, which after day 1, 4, 7, 11 of SAH as diameter of vasospasm artery measured at each time point. According to Liszczak's standard of evaluating the degree of vasospasm severity [4], vasospasm severity were calculated as: VSP $=$ (BAbase -BAmeasured) / BAbase $\times 100 \%$, where BAbase is the baseline diameter of the basilar artery and BAmeasured is 
the diameter of the basilar artery measured at a specific time. VSP=vasospasm severities percentage. We defined 3 categories of vasospasm severity: (1) minimal (vasospasm 10\%-20\%); (2) moderate (vasospasm 20\%-30\%); and (3) severe (vasospasm $\geq 30 \%$ ). The control of the contrast agent also accorded to the standard above to obtain the corresponding data. Morphology of basilar arteries were observed using VR technique.

Statistical Analyses. SPSS Version 11.0 (SPSS, Chicago, Ill) was used for analysis. All groups of data are expressed by mean \pm standard deviation $(\bar{x} \pm s)$. The measurement data were compared with single factor analysis of variance. Analysis of Ridit was used to compare the degree of vasospasm severity of basilar artery between animals before $\mathrm{SAH}$ in the control group and animals that after 4 days of SAH in the experimental group. With $\alpha=0.05$ as test level. P values of less than 0.05 were selected as the levels of significance.

\section{Results}

Measurements of MSCTA. All the images of the basilar artery of the 25 experiment rabbits were successfully obtained. The transverse diameter of the basilar artery that before SAH was 0.9 $1.7 \mathrm{~mm}$ ( mean $1.3 \mathrm{~mm}$ ) based on basilar artery image of the first MSCTA. Compared with the control group, change of diameter of the basilar artery that on day 1 after SAH was not significant $(P>0.05)$, however, there was a significant difference in basilar artery diameter between animals on day 4,7 , and 11 in the SAH group $(P<0.01)$, and the change was more apparent at the fourth day. (Fig. 1-5, Table 1); In the SAH group of days 4, 4/20 animals had no vasospasm, 4/20 animals had minimal vasospasm, 2/20 animals had moderate vasospasm, and 10/20 had severe vasospasm.(Table 2); There were two experiment rabbits happened to a second vasospasm in the SAH group. In the control group, 4/5 animals had no vasospasm, 1/5 animals had minimal vasospasm, no animals had moderate or severe vasospasm, while 1 of them happened to continued spasm after 11 days. In addition, change of basilar artery rough occurred in 2 animals (animals had no vasospasm in the control group) after 11 and 14 days.

Table 1: SAH group, diameter of basilar artery before and after SAH by VR $(\bar{x} \pm s, \mathrm{~mm})$

\begin{tabular}{ccc}
\hline Time $(\mathrm{d})$ & Number $(\mathrm{n})$ & Diameter $(\mathrm{mm})$ \\
\hline Before SAH & 5 & $1.30 \pm 0.40$ \\
Day 1 after SAH & 5 & $1.28 \pm 0.36^{\mathrm{a}}$ \\
Day4 after SAH & 5 & $0.82 \pm 0.24^{\mathrm{b}}$ \\
Day7 after SAH & 5 & $0.83 \pm 0.20^{\mathrm{b}}$ \\
Day 11 after SAH & 5 & $0.95 \pm 0.28^{\mathrm{b}}$ \\
\hline
\end{tabular}

Note: --- Single factor analysis of variance, a. Compare with the control group ( $\mathrm{P}>0.05)$; b. Compare with the control group $(\mathrm{P}<0.01)$.

Table 2: SAH group, Ridit analysis of basilar artery vasospasm severity before and after SAH on day 4

\begin{tabular}{|c|c|c|c|c|c|c|c|}
\hline \multirow[t]{2}{*}{ Groups } & \multicolumn{4}{|c|}{ vasospasm severity(number) } & \multicolumn{3}{|c|}{ Ridit analysis } \\
\hline & $<10 \%$ & $10 \% \sim$ & $20 \% \sim$ & $\geq 30 \%$ & Total & $\mathrm{R} \pm \mathrm{S}_{\mathrm{R}}^{-}$ & R95(confidence interval) \\
\hline $\begin{array}{l}\text { Before } \\
\text { SAH }\end{array}$ & 4 & 1 & 0 & 0 & 5 & $0.212 \pm 0.052$ & $0.1101 \sim 0.3139$ \\
\hline $\begin{array}{l}\text { After } \\
\text { SAH(4d) }\end{array}$ & 4 & 4 & 2 & 10 & 20 & $0.592 \pm 0.058$ & $0.4599 \sim 0.6863$ \\
\hline
\end{tabular}

Note:---P $<0.05$ 


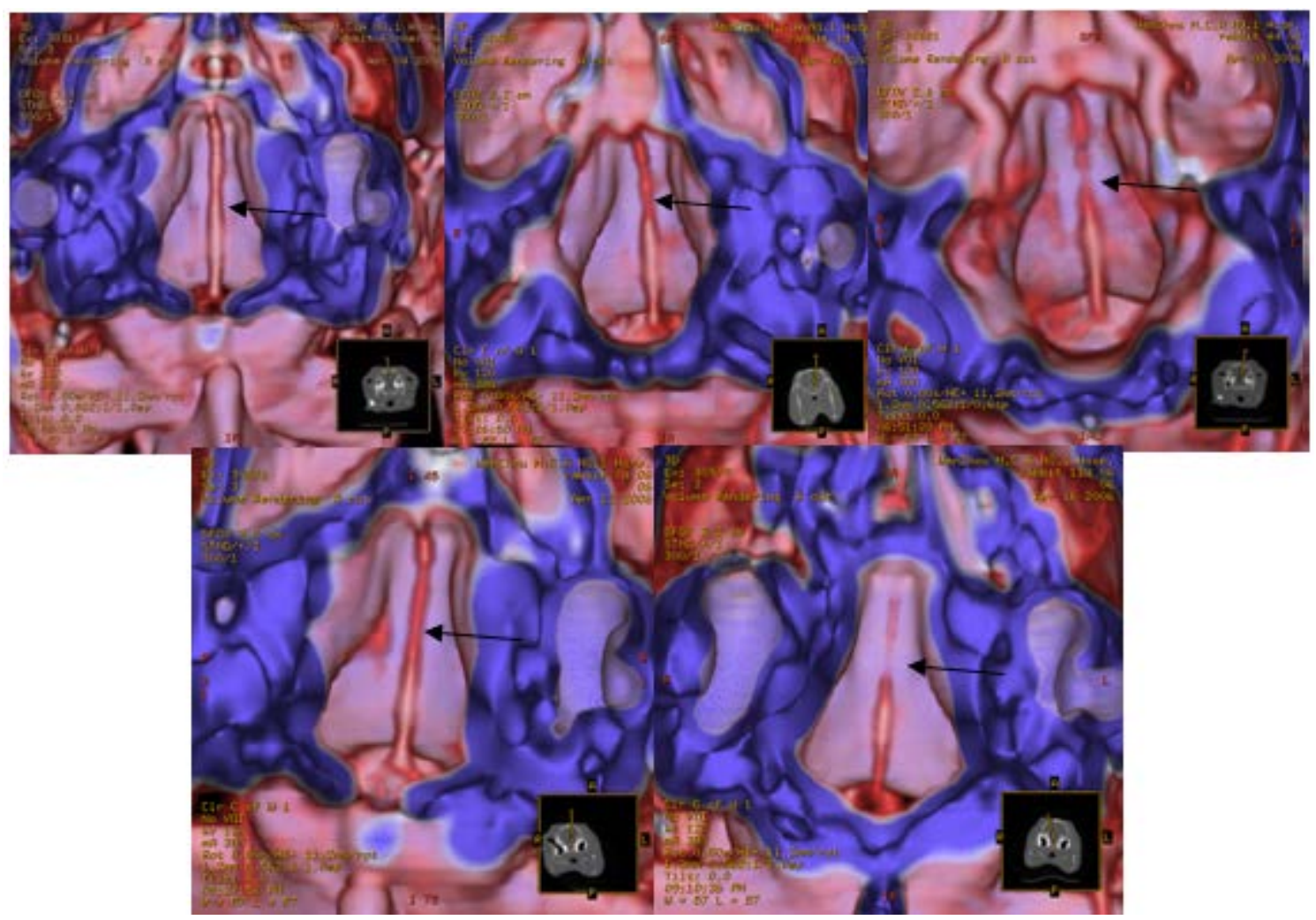

Fig. 1-5. SAH group , Imaging of rabbit basilar artery before SAH and day 1,4,7,11 after SAH

Fig.1 Display the normal basilar artery before blood injection(indicated by arrow above);

Fig.2 Day 1 after SAH, display mild narrowing at superior segment of basilar artery (indicated by arrow above);

Fig 3 Day 4 after SAH, display significantly narrowing(indicated by arrow above);

Fig.4 Day 7 after SAH, most of the artery recover(indicated by arrow above);

Fig. 5 Day 11 after SAH, occur a second vasospasm(indicated by arrow above).

Animal Behavior and Neurological Status. Most of the experiment rabbits in the SAH group developed the eye tremor levels and rabid breathing immediately after first injection of blood. The rabbits showed mental fatigue, drowsiness, spontaneous activity decreasing, and so was the feeding and drinking. They would react to the normal stimulation. Symptoms increased significantly after double injection of blood. Two rabbits appeared to mental fatigue, feeding and drinking decreasing in the control group $(n=5)$. However, 3 of them had not obviously change in such aspect: neurological status and feeding.

\section{Discussion}

CVS the serious complication of SAH of which incidence is as high as 30\%-90\% [1, 7-13] and $50 \%$ of the patients with angiographic vasospasm will eventually develop ischemic neurologic deficits [14]. Delayed cerebral vasospasm that in large blood vessels of brain parenchyma outside occurs in approximately 70\% of SAH survivors after hemorrhage for 4-7days, and the mortality it causes is even more than that of aneurysm rupture and rebleeding [9,15] $40 \%$ of the patients has no symptoms, while $30 \%$ of survivors remain the persistent cognitive impairment and focal neurological deficits[10,16]. As a result, it becomes a major cause of death and severe disability. Hence for years, people have been working on pathology and physiology animal experiment of CVS after SAH. At present, the animal model of SAH has already been very mature, but the evaluation methodology of CVS had limitations in different degree. By getting tissue from animal for pathological examination and measuring diameters of the vessels will directly affect the accuracy of the result, as the vessel may get changed during the course of killing animal. Moreover, the experiment animal is not recycled.TCD could indirectly detect the spastic of rabbit basilar artery by observing the speed of blood, but data stability would be limited. Though DSA is regarded as the gold standard assessment for CVS, it is complex and difficult to small animals, like rabbit etc [5-6]. 
What is more, the same animal is difficult to be done repeated examinations over the long time and the change of vasospasm could not be observed dynamically in vivo with a high-mortality. Consequently, a more convenient, stable and repeatable method is required to do the animal experiment research. According to the result of our study, the success rate of basilar artery imaging was $100 \%$, and the rabbits were all alive during the examination by MSCTA. It shows that the technique is reliable, and its greatest advantage is that we can observe the change of vasospasm dynamically in vivo for the same animal.

20 rabbits in the SAH group had been done five examinations by MSCTA before SAH and 1, 4, 7 and 11 days after SAH. According to Liszczak's standard [4], diameter of basilar artery before $\mathrm{SAH}$ was defined as the baseline, and then the corresponding diameter after SAH could be used to compare with that before SAH. The results of statistical analyses showed that compared with the time, before $\mathrm{SAH}$, other time points (except for day 1 ) were significant different $(\mathrm{P}<.01)$, day 4 and 7 were more apparent. There were differences among day 4, 7 and 11, but this difference was not significant $(\mathrm{P}>0.05)$. This accorded with pathological process of vessel morphological change after vasospasm [11, 17] because the peak of SAH patient with CVS often occurred on day 4-14 after bleeding [12, 18-19].

Our experiment used VR to measure the rabbit basilar artery. It is VR that gives a particular CT voxel corresponding color, brightness, contrast and transparency, artificially changing the brightness and contrast. Hence, it can change the contrast between the surrounding and tissue without distortion, highlight the shape of the target, and retain all of the original data of the fault. So that three levels of the target is richer and form becomes vivid and accurate. In the present study, the mean diameter of basilar artery was $1.30 \mathrm{~mm}$, which approach to $1.1 \mathrm{~mm}$ measured by selective vertebra-basilar angiography [1].

It's worth noting that in the control group of our experiment rabbits, one in the late occurred moderate vasospasm and lasted to day 11 . Whether contrast agents have an impact on the blood vessels or not, there are different views on the literature. Luo's research [20] indicated that after saline, $70 \%$ diatrizoate, or Omnipaque or autologous blood plus Omnipaque was injected into subarachnoid of the normal and cerebral vasospasm rabbit, each group of intracranial internal carotid artery blood flow velocity was no significant difference, and DSA examination was normal. In addition, there was also no significant difference if Omnipaque was injected into subarachnoid after CVS. Therefore, different types of contrast agents do not cause or aggravate cerebral vasospasm. However, Juni et al. [21] held that some patients would result in CVS during brain angiography by using Onipaque. Minuk et al. [22] agreed with that contrast agents would make endothelial cells shrink, then change the stability of blood-brain barrier and tight junctions of endothelial cell. Consequently, contrast agent got into the brain nerve tissue gap, caused a direct toxic effect. Unlike the result of Luo zhiyi's experiment [20], intravascular injection of contrast agent could lead to CVS in our research. To Juni [21] and Minuk [22] description, CVS resulted from intravascular injection of non-ionic contrast agents. Whether the studies have certain comparable and correlation remains to be seen. Furthermore, in our experiment, change of basilar artery rough occurred in 2 animals (animals had no vasospasm in the control group) after 11 and 14 days and 1 of them happened to continued spasm after 11 days, two happened to a second vasospasm in the SAH group. Whether the appearances are related to repeated injection of contrast agent, is still unknown. In conclusion, our study may be limited by small samples of control group, the direct relationship between contrast agents and CVS as well as the indirect relationship among other factor cannot be determined. That is, more experiments still have to be established in future.

\section{Acknowledgement}

This study was supported, in part, by the National Key Project of Scientific and Technical Supporting Program funded by the Ministry of Science and Technology of China during the 12th Five-Year Plan (Project No. 2011-BAI08B09). The Wenzhou Municipal Science and Technology Project (Y201400290) and Nature Science Foundation of Zhejiang Province (Project No. 
LQ15H180002) .

\section{References}

[1] Shi ZS, Huang ZS, Guo SL, et al., The experimental study for selective vertebra-basilar arteriography in rabbits, Chin J Exp Surg. 21 (2004) 766-766.

[2] Megyesi JF, Vollrath B, Cook DA, et al., In vivo animal models of cerebral vasosp asm: a review, Neurosurgery. 46 (2000) 448-461.

[3] Bowman, George, Dixit, Sanat, Bonneau, Robert H.,Bowman G, Dixit S, Bonneau RH, et al., Neutralizing antibody against interleukin-6 attenuates post hemorrhagic vasospasm in the rat femoral artery model, Neurosurgery. 54 (2004):719-726.

[4] Liszczak T M, Varsos V G, Black P M L, et al. , Cerebral arterial constriction after experimental subarachnoid hemorrhage is associated with blood components within the arterial wall, J Neurosurg. 58 (1983) 18-26.

[5] Wang Y, Zhong M, Tan XX, et al., Detection methods of rabbit basilar artery CVS model by injection of autologous arterial blood into the cisterna rnagna twice, Chin J Exp Surg. 24 (2007) 375.

[6] Zhu XL, Ding Y, Liu YZ, et al., Imaging study of cerebral ischemic model in rabbits, Chinese Journal of Radiology. 33 (1999) 168-170.

[7] Ye W, Jiang CL, Li YL, et al., The experimental study for vasospasm of rabbit basilar artery, Chin J Neurosurg. 17 (2001) 178-180.

[8] Helbok R, Kurtz P, Vibbert M, et al., Early neurological deterioration after subarachnoid haemorhage: risk factors and impact on outcome, J Neurosurg Psychiatry. 84 (2013) 266-270.

[9] Crowley R W, Medel R, Dumont A S, et al., A ngiographic vasospasm is strongly correlated with cerebral in farction after sub-arachnoid hemorrhage, Stroke. 42 (2011) 919- 923.

[10] Frontera J A, Ahmed W, Zach V, et al., Acute ischaemia after subarachnoid haemorrhage, relationship with early brain injury and impact on outcome: a prospective quantitative MRI study, Journal of Neurology, Neurosurgery \& Psychiatry. 2014: jnnp-2013-307313.

[11] Raslan F, Albert-Weissenberger C, Westermaier T, Sa-ker S, Kleinschnitz C, Lee J Y, A modified double injection model of cisterna magna for the study of delayed cerebral vasospasm following subarachnoid hemorrhage in rats, Exp Transl Stroke Med. 4 (2012) 23.

[12] Kim J H, Yi H J, Ko Y, et al., Effectiveness of papaverine cisternal irrigation for cerebral vasospasm after aneurysmal subarachnoid hemorrhage and measurement of biomarkers, Neurological Sciences. 35(2014) 715-722.

[13] Hou J, Zhang JH, Does prevension of vasospasmin subarachnoid hemorrhage improve clinical outcome? No, Strock. 44 (2013) S34- S36.

[14] Macdonald RL, J, Zhang JH., Does prevension of vasospasmin subarachnoid hemorrhage improve clinical outcome? Yes, Strock. 44 (2013) S31- S33.

[15] Wintermark M, Ko NU, Smith WS, et al., Vasospasm after subarachnoid:hemorrh age :utility of perfusion CT angiography on diagnosis and management, Am J Neuroradial. 27 (2006) 26-34.

[16] Zhang JZ,Long LS,Han RZ, et al., Recent advance in cerebrovascular pathological changes induced by subarachnoid hemorrhage, Chin J Neuromed. 9 （2010）1291-1293.

[17] Jeon H, Ai J, Sabri M, et al., Neurological and neurobehavioral assessment of experimental subarachnoid hemorrhage, BMC Neurosci. 10 (2009) 103.

[18] Zhou ML,Shi JX,Zhu JQ, et al., Comparison between one- and two-hemorrhage models of 
cerebral vasospasm in rabbits, J Neurosci Methods. 159 (2007) 318-324.

[19] Wang QS,Li G., Recent advance in pathogenesis of cerebral vasospasm after subarachnoid hemorrhage, Chin J Neuromed. 12 (2013) 422-425.

[20] Luo ZY,Ma LT,Wu ZQ., Recent advance in pathogenesis of cerebral vasospasm after subarachnoid hemorrhage, Chin J clin Neurosurg. 7 (2002) 110-112.

[21] Juni J , Morare JM , Lainez J,Juni J, Morera J, Láinez JM, et al., Transient global amnesia after cerebral angiography with iohexol, Neruoradiology. 34 (1992) 141-143.

[22] Minick J , Melancon D, Tampieir D ,Minuk J, Melançon D, Tampieri D,et al., Transient global amnesia associated with cerebral angiography performed with use of iopamidol, Radiology. 17 (1990) 285-286. 